Vol. 43 (1991) [19-36]

\title{
HIGHLY TRANSITIVE REPRESENTATIONS OF FREE GROUPS AND FREE PRODUCTS
}

\author{
A.M.W. Glass and Stephen H. McCleary \\ Dedicated to B.H. Neumann on his 80 th birthday \\ with our appreciation and respect.
}

\begin{abstract}
A permutation group is highly transitive if it is n-transitive for every positive integer $n$. A group $G$ of order-preserving permutations of the rational line $Q$ is highly order-transitive if for every $\alpha_{1}<\ldots<\alpha_{n}$ and $\beta_{1}<\ldots<\beta_{n}$ in $Q$ there exists $g \in G$ such that $\alpha_{i} g=\beta_{i}, i=1, \ldots, n$. The free group $F_{\eta}\left(2 \leqslant \eta \leqslant \aleph_{0}\right)$ can be faithfully represented as a highly order-transitive group of order-preserving permutations of $Q$, and also (reproving a theorem of $\mathrm{McDonough}$ ) as a highly transitive group on the natural numbers $N$. If $G$ and $H$ are nontrivial countable groups having faithful representations as groups of order-preserving permutations of $\mathbf{Q}$, then their free product $G * H$ has such a representation which in addition is highly order-transitive. If $G$ and $H$ are nontrivial finite or countable groups and if $H$ has an element of infinite order, then $G * H$ can be faithfully represented as a highly transitive group on $N$. Some of the representations of $F_{\eta}$ on $\mathbf{Q}$ can be extended to faithful representations of the free lattice-ordered group $L_{\eta}$.
\end{abstract}

\section{INTRODUCTION}

Our main results are

Theorem A. The free group $F_{\eta}\left(2 \leqslant \eta \leqslant \aleph_{0}\right)$ can be faithfully represented as a highly order-transitive group $\widehat{F}_{\eta}$ of order-preserving permutations of the rational line Q.

ThEOREM B. (McDonough [10]). The free group $F_{\eta}\left(2 \leqslant \eta \leqslant \aleph_{0}\right)$ can be faithfully represented as a highly transitive group of permutations of the natural numbers N.

In connection with the next theorem, we mention that a countable group $G$ can be embedded in $A(Q)$ if and only if $G$ can be right ordered; see Section 4.

Received 30 January 1990

We wish to thank P.J. Cameron for his lectures at the Durham Conference, "Model Theory and Groups, 1988"; they provided the impetus for this research. The first author is most grateful to the London Mathematical Society for funding to attend the conference, and to the organisers Wilfrid Hodges and Peter M. Neumann for providing such a stimulating program; he also expresses his appreciation for partial funding provided for this research by the Bowling Green Faculty Research Committee.

Copyright Clearance Centre, Inc. Serial-fee code: 0004-9729/91 \$A2.00+0.00. 
TheOREM C. Let $G$ and $H$ be nontrivial countable groups. If $G$ and $H$ have faithful representations as groups of order-preserving permutations of $Q$, then their free product $G * H$ has such a representation which in addition is highly order-transitive.

THEOREM D. Let $G$ and $H$ be nontrivial finite or countable groups, with $H$ having an element of infinite order. Then their free product $G * H$ can be faithfully represented as a highly transitive group of permutations of the natural numbers $N$.

As we shall see in Section 5 , the conclusion of Theorem $D$ fails for $Z_{2} * Z_{2}$, where $Z_{2}$ denotes the cyclic group of 2 elements.

QUESTION. For which non-trivial groups $G$ and $H$ does $G * H$ have a faithful highly transitive representation on $\mathrm{N}$ ?

THEOREM E. Every faithful representation of $F_{\eta}\left(2 \leqslant \eta<\aleph_{0}\right)$ as a highly ordertransitive group of order-preserving permutations of $Q$ (respectively as a highly transitive group of permutations of $N$ ) can be extended to such a representation of $F_{\aleph_{0}}$.

McDonough [10] and Dixon [1] have given quite different proofs of Theorem $B$. Here we prove Theorem A, its analogue for $Q$, and find ourselves with yet another proof of Theorem B. (As an aside, we mention that analogues of Dixon's other results in [1] also hold.) Another way of stating these results is to say that $F_{\eta}$ can be densely embedded in the group $A(Q)$ of ordering-preserving permutations of $Q$, and can be densely embedded in $\operatorname{Sym}(N)$. (A subgroup $H$ of a permutation group $(G, \Omega)$ is dense if whenever $\alpha_{i} g=\beta_{i}(i=1, \ldots, n)$ for some $g \in G$, then also $\alpha_{i} h=\beta_{i}(i=1, \ldots, n)$ for some $h \in H$.)

The proofs of Theroems A and B are prerequisites for the more intricate proofs of their generalisations Theorems $\mathrm{C}$ and $\mathrm{D}$. However, the proofs of $\mathrm{C}$ and $\mathrm{D}$ may be read in either order and do not require Section 3.

On a different historical track, the present authors obtained results about free lattice-ordered groups similar to those above. The present techniques mostly stem from the lattice-ordered group ( $\ell$-group) proofs. However, no knowledge of $\ell$-groups is needed except in the last section, where new results about free $\ell$-groups are to be found.

\section{FREE GROUPS}

First, a bit of background. $F_{\eta}$ can be made into a totally ordered group ( $a \leqslant b$ implies cad $\leqslant c b d$ for all $a, b, c, d$ ) which is dense in itself [2, Chapter IV, Theorem 8]. Also, every chain (that is, every totally ordered set) which is countable, dense in itself, and lacks end points (for example, $F_{\eta}$ ordered as above) is order-isomorphic to the rational line $Q$.

Let $\mathbf{x}$ be a fixed set of free generators for $F_{\eta}$. Our fundamental tool will be the notion of a diagram for a reduced group word $w=x_{i_{1}}^{ \pm 1} \ldots x_{i_{n}}^{ \pm 1}\left(x_{i_{j}} \in \mathbf{x}\right)$. As an 
example, consider $w=x_{1} x_{2} x_{1}^{-1} x_{2} x_{1}$, and a substitution in $A(Q)$ moving 0 to some $\delta \neq 0$ :

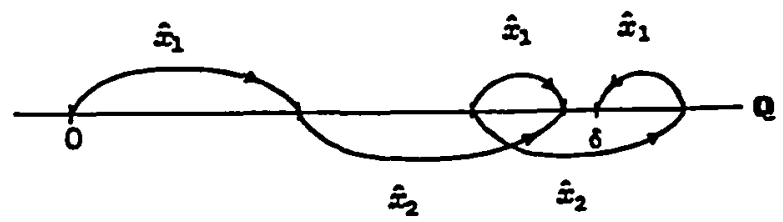

Figure 1. A diagram.

This diagram shows that (to no one's surprise) $w \neq e$ in $F_{\eta}$. The precise definition (making no reference to $Q$ ) is as follows:

The points of the diagram are the initial subwords $x_{i_{1}}^{ \pm 1} \ldots x_{i_{k}}^{ \pm 1}(0 \leqslant k \leqslant n)$ of $w$. For each ordered pair $(\alpha, \beta)$ of points such that $\alpha x_{i_{j}}^{ \pm 1}=\beta$, the diagram includes an $x_{i_{j}}$-arrow, from $\alpha$ to $\beta$ if the exponent on $x_{i_{j}}$ is +1 , otherwise from $\beta$ to $\alpha$. The remaining aspect of the diagram is a total order on the set $\Delta$ of points which is consistent with the arrows in that if there are $x$-arrows from $\alpha_{1}$ to $\beta_{1}$ and $\alpha_{2}$ to $\beta_{2}$ (same $x$ for both), then $\alpha_{1} \leqslant \alpha_{2}$ if and only if $\beta_{1} \leqslant \beta_{2}$. (An $x$-arrow from $\alpha$ to $\beta$ may alternately be described as an $x^{-1}$-arrow from $\beta$ to $\alpha$.) The empty initial subword $(k=0)$ is called the base point of the diagram.

By a diagram on $Q$ we mean a diagram which arises from a substitution in $A(Q)$.

LEMmA 1. Let $w \in F_{\eta}$. If $w \neq e$, there exists a diagram on $Q$ showing this (by making $0 \widehat{w} \neq 0$ ). Moreover, given any diagram for $w$ drawn on $Q$, there is for each $x \in \mathbf{x}$ an order-preserving permutation $\widehat{x}$ of $\mathbf{Q}$ which acts in accordance with all the $x$-arrows.

Proof: For the first claim, make $F_{\eta}$ into a totally ordered group isomorphic as a chain to $Q$, and use its right regular representation. For the second, the constraints imposed by the collection of $\mathbf{x}$-arrows can be simultaneously satisfied by some $\widehat{x} \in A(\mathbf{Q})$ because all open rational intervals are isomorphic as chains.

Proof of Theorem A: For each $x \in \mathrm{x}$, the action on $\mathrm{Q}$ of its image $\widehat{x}$ will be specified at enough points to guarantee the desired results. Each specifiction will amount to an $\boldsymbol{x}$-arrow. The proof splits into three distinct phases:

(1) Specifications (essentially within $Q^{+}$, the positive rationals) to achieve faithfulness and to link $\mathbf{Q}^{+}$with $\mathbf{Q}^{-}$by arranging that every $n$-tuple in $Q$ can be sent to an $n$-tuple in $\mathbf{Q}^{-}$.

(2) Specifications within $Q^{-}$to achieve high order-transitivity.

(3) Synthesis, in which for each $x \in \mathbf{x}$ we choose an order-preserving permutation $\widehat{x}$ of $Q$ which meets the specifications for $\widehat{x}$. 
FAIThFulness AND LINKAgE. $F_{\eta}$ is countable, and we enumerate its nonidentity elements as $w_{0}, w_{1}, \ldots$ In the rational interval $[0,1]$, we lay out a copy of a diagram for $w_{0}=x_{i_{1}}^{ \pm 1} \ldots x_{i_{n}}^{ \pm 1}$ showing $e \neq w_{0}$, with the smallest point $\lambda_{0}$ of the diagram taken to be 0 , and the largest point $\rho_{0}$ taken to be 1 . We specify about the $\widehat{x}$ 's that the point (corresponding to) $x_{i_{1}}^{ \pm 1} \ldots x_{i_{k-1}}^{ \pm 1}$ be sent by $\widehat{x}_{i_{k}}^{ \pm 1}$ to the point (corresponding to) $x_{i_{1}}^{ \pm 1} \ldots x_{i_{k-1}}^{ \pm 1} x_{i_{k}}^{ \pm 1}$. Similarly, in each interval $[2 n, 2 n+1]$, we lay out such a diagram for $w_{n}$ and make such specifications. This is already enough to give faithfulness.

For the sake of the linkage, we want to ensure that all the points in the various diagrams lie in the same orbit of $\widehat{F}_{\eta}$, and this orbit extends down into $Q^{-}$. Then because the diagram points are cofinal in $Q$ and all permutations in $\widehat{F}_{\eta}$ will preserve order, we will have the desired linkage.

To make all diagram points lie in one orbit, it suffices to arrange that for each $n=0,1, \ldots$, the points $2 n+1$ and $2(n+1)$ lie in the same orbit. For this we construct appropriate "bridges".

We begin with the interval $[1,2]$. In the original diagram for $w_{0}, \rho_{0}(\leftrightarrow 1)$ must have been moved by at least one free generator, say $x_{\lambda_{0}}$; and in the diagram for $w_{1}$, $\lambda_{1}(\leftrightarrow 2)$ must have been moved by some free generator $x_{\lambda_{1}}$. We decree that

(a, $1 \widehat{x}_{\rho_{0}}=4 / 3$ if $\rho_{0}$ was moved up by $x_{\rho_{0}}$,

(a $) \quad 1 \widehat{x}_{\rho_{0}}^{-1}=4 / 3$ if $\rho_{0}$ was moved down by $x_{\rho_{0}}$,

(b1 $)(5 / 3) \widehat{x}_{\lambda_{1}}=2$ if $\lambda_{1}$ was moved up by $x_{\lambda_{1}}$,

$\left(b_{2}\right)(5 / 3) \widehat{x}_{\lambda_{1}}^{-1}=2$ if $\lambda_{1}$ was moved down by $x_{\lambda_{1}}$.

To connect $4 / 3$ and $5 / 3$, we further decree that $(4 / 3) \widehat{x}_{\rho_{0}}=5 / 3$ if $\left(a_{1}\right)$ obtains (or that $(4 / 3) \widehat{x}_{\rho_{0}}^{-1}=5 / 3$ if $\left(a_{2}\right)$ obtains); except that this may conflict with $\left(b_{2}\right)$ or $\left(b_{1}\right)$ if $x_{p_{0}}=x_{\lambda_{1}}$, so in that case we pick any other $x \in \mathrm{x}(\eta>1)$ and decree that $(4 / 3) \widehat{x}=$ $5 / 3$. We build similar bridges in the other intervals $[2 n+1,2 n+2], n=1,2, \ldots$

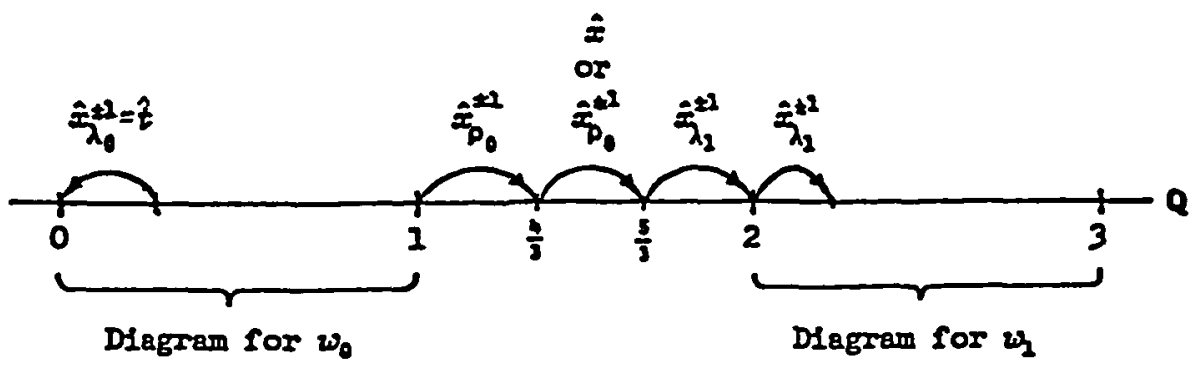

Figure 2

Replacing $x_{\lambda_{0}}$ by $x_{\lambda_{0}}^{-1}$ if necessary, we may assume $\widehat{x}_{\lambda_{0}}$ moves some positive point to 0 . We denote $x_{\lambda_{0}}$ by $t$. Thus the figure contains a leftward $t$-arrow with head at 0 . 
We specify that $\alpha \hat{t}=\alpha-1$ for all $\alpha \leqslant 0$. Now the orbit of $\widehat{F}_{\eta}$ containing the diagram points extends down into $Q^{-}$, and we have linkage.

Note. White's Theorem [12] that the real maps $\alpha \mapsto \alpha+1$ and $\alpha \mapsto \alpha^{3}$ generate a free group fixing no transcendental real number could have been used to accomplish faithfulness and linkage; see the proof of Theorem A3. However, that approach would not carry over to free products.

HIGH ORDER-TRANSITIVITY. We fix $b \in \mathbf{x}$ with $b \neq t$, and specify that $0 \widehat{b}=0$. Let $\mathcal{S}_{n}$ be the set of pairs $\left(\alpha_{1}, \ldots, \alpha_{n}\right),\left(\beta_{1}, \ldots, \beta_{n}\right)$ of strictly negative rationals with $\alpha_{1}<\ldots<\alpha_{n}$ and $\beta_{1}<\ldots<\beta_{n}$. Enumerate $\mathcal{S}=\bigcup_{n=1}^{\infty} \mathcal{S}_{n}$.

For the first pair $\left(\alpha_{1}, \ldots, \alpha_{n_{1}}\right),\left(\beta_{1}, \ldots, \beta_{n_{1}}\right)$, we specify that $\alpha_{i} \widehat{b}=\beta_{i}$ $\left(i=1, \ldots, n_{1}\right)$; see Figure 3. Next, we pick a (negative) integer $m_{1}<\min \left\{\alpha_{1}, \beta_{1}\right\}$, and specify that $m_{1} \widehat{b}=m_{1}$. For the next pair $\left(\mu_{1}, \ldots, \mu_{n_{2}}\right),\left(\mu_{1}, \ldots, \mu_{n_{2}}\right)$, we specify that $\left(\mu_{i}+m_{1}\right) \widehat{b}=\nu_{i}+m_{1}\left(i=1, \ldots, n_{2}\right)$, which entails no conflict with any previous specification, thus arranging that $\mu_{i} \widehat{t}^{-m_{1}} \widehat{b} \bar{t}^{m_{1}}=\nu_{i}\left(i=1, \ldots, n_{2}\right)$. Next, we pick an integer $m_{2}<\min \left\{\mu_{1}+m_{1}, \nu_{1}+m_{1}\right\}$, and specify that $m_{2} \widehat{b}=m_{2}$. For the third pair $\left(\sigma_{1}, \ldots, \sigma_{n_{3}}\right),\left(\tau_{1}, \ldots, \tau_{n_{3}}\right)$, we specify that $\left(\sigma_{i}+m_{2}\right) \widehat{b}=\tau_{i}+m_{2} \quad\left(i=1, \ldots, n_{3}\right)$, and we pick an integer $m_{3}<\min \left\{\sigma_{1}+m_{2}, \tau_{1}+m_{2}\right\}$ and specify that $m_{3} \widehat{b}=m_{3}$. Continuing in this manner, we arrange that every negative $n$-tuple can be sent to every other negative $n$-tuple (same $n$ ) by some $\hat{t}^{-m} \widehat{b}^{m}$.

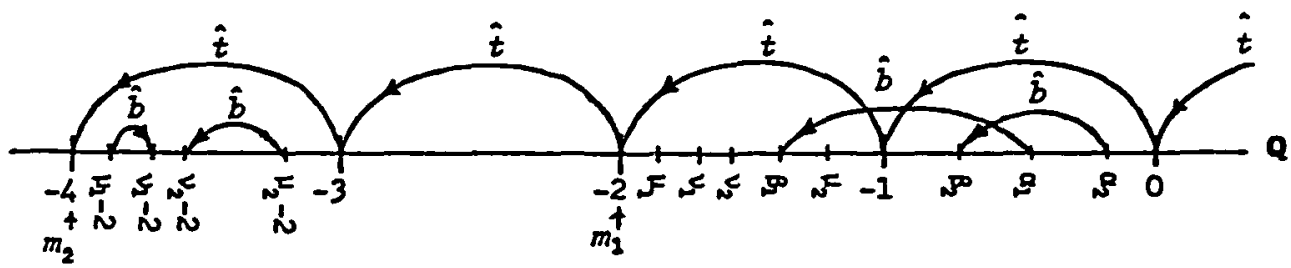

Figure 3

SYNTHESIS. For each $x \in \mathbf{x}$, the set of points which are heads of specified $x$-arrows is cofinal (coinitial) in $Q$ if and only if the same holds for tails. For each $x$ other than $t$, the set of points which are ends of specified $x$-arrows has no limit points in $R$, and for $t$ only the nonpositive reals are limit points. Hence we can extend each $\widehat{x}$ to an order-preserving permutation of $\boldsymbol{Q}$.

Proof of Theorem B: Establish a one-to-one correspondence between $\mathbf{N}$ and Q. Then proceed as in the proof of Theorem $A$, but this time in the proof of high order-transitivity include all negative $n$-tuples $\left(\alpha_{1}, \ldots, \alpha_{n}\right)$ of distinct points without 
requiring $\alpha_{1}<\cdots<\alpha_{n}$. Each $\widehat{x}$ except for $\widehat{b}$ preserves order, and even $\widehat{b}$ preserves order on the nonnegative rationals and maps the set of nonnegative rationals onto itself. Thus again we have the desired linkage.

THEOREM AB2. Theorem $A$ holds for $F_{\eta}$ of any infinite rank, except that $Q$ must be replaced by a suitable chain $\Omega_{\eta}$; and similarly for Theorem $B$.

The proof is considerably easier than for finite rank, and is almost identical to the proof for free lattice-ordered groups; see [7, Theorem 1].

\section{Sharpening THE CONCLUSIONS FOR FREE GROUPS}

The preceding proofs pave the way for the proofs of our more general results about free products. However, we digress now to look at variations of the free group proofs which give slightly sharper conclusions. Obviously it can happen in the proof of Theorem $A$ that $\widehat{b}$ fixes all points in some interval. In the opposite spirit is:

Theorem A3. In Theorem $A$, it can be arranged that for every $e \neq w \in F_{\eta}$, there exists $q_{w} \in \mathbf{Q}$ such that $\widehat{w}$ moves all irrationals above $q_{w}$.

ProOF: Faithfulness and linkage can be achieved directly by White's Theorem [12] that the maps $\alpha f=\alpha+1$ and $\alpha g=\alpha^{3}$ freely generate a copy $\widehat{F}_{2}$ of $F_{2}$ within $A(\mathbf{R})$, and that indeed every $e \neq \widehat{w} \in \widehat{F}_{2}$ moves every transcendental number.

We have $\widehat{F}_{2} \leqslant A(\mathrm{~A})$, where $\mathrm{A}$ denotes the real algebraic numbers. Let $f_{i}=g^{-i} f g^{i}$, $i=0,1, \ldots$ Let $\widehat{k}_{i}=\widehat{f}_{i}$, but change $\widehat{k}_{2}$ to the left of 0 to make $\left\langle\widehat{k}_{1}, \widehat{k}_{2}\right\rangle$ highly ordertransitive. Now use the copy of $\widehat{F}_{\eta}$ freely generated by $\left\{\widehat{k}_{i} \mid 0 \leqslant i<\eta\right\}$. This gives Theorem A3 with "irrationals" changed to "transcendentals". Since the chains $A$ and $Q$ are isomorphic making the chain of real transcendentals and the chain of irrationals isomorphic, the theorem follows.

Along the same lines is

ThEOREM A4. Let $T$ be any countable dense subset of the irrationals. In Theorem $A$, it can be arranged that $T$ be an orbit of $\widehat{F}_{\eta}$ and that every $e \neq \widehat{w} \in F_{\eta}$ move every point in $T$.

Proof: This time we change the proof of Theorem A by changing the first part, with an argument modelled after the proof of $[8$, Theorem 3$]$.

For any $T_{1}$ and $T_{2}$ satisfying our hypotheses, there is an order-preserving permutation of $Q$ sending $T_{1}$ onto $T_{2}[6$, Lemma 21]. Thus we may assume that $T$ is a coset of $Q$ in $(R,+)$, so that $T$ is mapped onto itself by integer translations.

As before, let $\alpha \widehat{t}=\alpha-1$, specify that $0 \widehat{b}=0$, and make specifications for $\widehat{b}$ in $Q^{-}$to arrange high order-transitivity. These specifications amount to a collection of $\widehat{b}$-arrows, and more $\widehat{b}$-arrows will be added later. Let $B \subseteq \mathbf{Q}^{-}$be the set of points that are ends of $\widehat{b}$-arrows. $\mathcal{B}$ has no limit points in $R$. 
For faithfulness, we use a brute force argument quite different from that of Theorem $A$. Let $y$ be $x$ with $t$ deleted. Let $\mathcal{P}$ be the set of pairs $(\alpha, z)$, where $\alpha \in Q \cup T$ and $z=y^{ \pm 1}$ for some $y \in \mathbf{y}$. We proceed inductively through the enumeration, defining $\hat{y}$-arrows $(y \in \mathbf{y})$ as we go. There are two cases: $z=y$ and $z=y^{-1}$.

When we reach $(\alpha, y)$, we specify a $\hat{y}$-arrow with tail at $\alpha$ (unless there is already such an arrow). Now we explain how to choose the head $\beta$ of this arrow. Let $\lambda$ be the largest point in $Q \cup T$ which is the head of an already existing $\widehat{y}$-arrow whose tail lies below $\alpha$; or if there aren't any, let $\lambda=-\infty$. (Only finitely many $\hat{y}$-arrows have been added so far during the induction, and by the construction of $\widehat{b}$-arrows prior to the induction there must be a largest such point, even for $y=b$.) Let $\rho$ be the smallest of the points that are heads of already existing $\widehat{y}$-arrows whose tails lie above $\alpha$; or if there aren't any, let $\rho=+\infty$. We choose the head $\beta$ of the new $\hat{y}$-arrow from $(\lambda, \rho)$, and from the same set $Q$ or $T$ as $\alpha$.

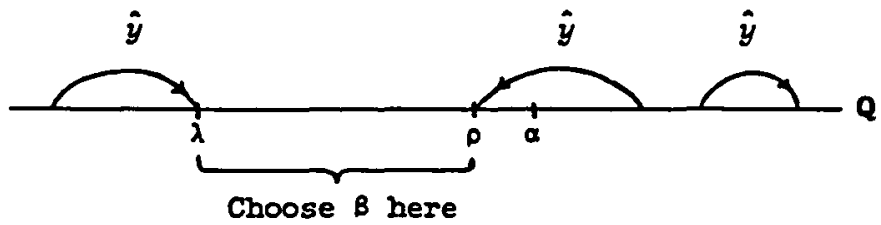

Figure 4

If $\alpha \in T$, we impose one more constraint on $\beta$, namely that $\beta$ not differ by an integer from $\alpha$ or from either end of any previously defined $\widehat{u}$-arrow $(u \in y)$. (The $\widehat{b}$-arrows specified prior to the induction have their ends in $Q$, so there are only finitely many ends of $\widehat{u}$-arrows in $T$.) The reason for the constraint is this: A loop is a path which starts at some $\delta \in Q \cup T$ and follows a sequence of arrows ( $(\hat{t}$-arrows are included here) and eventually returns to $\delta$. (No arrow has its two ends the same, removing any ambiguity from this definition.) The constraint makes sure that no sequence of $\hat{t}$-arrows leads from $\beta$ to $\alpha$ or to any point which is either end of an already existing $\widehat{u}$-arrow $(u \in y)$. This in turn guarantees that as we proceed through the induction, there can never arise for the first time a loop involving a point from $T$. And this guarantees that no $\widehat{w} \neq e$ fixes any point in $T$ (which then guarantees faithfulness).

We treat the case $z=y^{-1}$ similarly, this time defining a $\widetilde{y}^{-1}$-arrow from $\alpha$ to $\beta$ (that is, a $\hat{y}$-arrow from $\beta$ to $\alpha$ ).

The $\hat{y}$-arrows defined prior to and during the induction define an order preserving permutation of $Q$ (onto $Q$ because of the case $z=y^{-1}$ ) which maps $T$ onto itself.

In all the preceding proofs, every nonidentity element of $\widehat{F}_{\eta}$ has unbounded support. This is no accident: 
Proposition 2. F cannot be faithfully represented as a transitive group of order-preserving permutations of a chain $Q$ so as to include both a nonidentity element whose support is bounded above and another whose support is bounded below.

Proof: Suppose $\widehat{g}_{A}$ has support bounded above and $\widehat{g}_{B}$ has support bounded below. By transitivity, $\operatorname{supp}\left(\widehat{g}_{A}\right)<\operatorname{supp}\left(\hat{f}^{-1} \widehat{g}_{B} \hat{f}\right)$ for some $f \in G$. Hence $\hat{f}^{-1} \widehat{g}_{B} \hat{f}$ commutes with $\hat{g}_{A}$, and so has a common root with $\widehat{g}_{A}$; this makes their supports coincide, which is a contradiction.

Proof of Theorem E: We prove the $Q$ version, the $N$ version being similar. Our task is to specify $\widehat{x}_{\eta+1}, \widehat{x}_{\eta+2}, \ldots$ so that the resulting representation of $\widehat{F}_{\mathbb{K}_{0}}$ on $\mathbf{Q}$ is faithful. By induction, it suffices to specify just $\widehat{x}_{\eta+1}$, which we denote by $\widehat{y}$.

We enumerate the elements of $\widehat{F}_{\eta+1} \backslash \widehat{F}_{\eta}$ as $w_{0}, w_{1}, \ldots$ Suppose by induction that we have specified a finite number of $\widehat{y}$-arrows which together with the given $\widehat{x}_{1-}, \ldots, \widehat{x}_{\eta}$ arrows create for each of $i=0, \ldots, k-1$ a diagram showing $w_{i} \neq e$. Pick $\beta=\beta_{k} \in Q$ exceeding both ends of all these $\widehat{y}$-arrows. Let $w^{*}$ be the longest final subword of $w_{k}$ which involves no $y^{ \pm 1}$ 's. Pick $\delta>\beta \widehat{w}^{*}$. We will arrange that $\delta \widehat{w}_{k} \neq \delta$. Beginning at $\delta$, we trace through $w_{k}$, noting the $\widehat{x}_{1^{-}}, \ldots, \widehat{x}_{\eta}$-arrows which arise and specifying heads of $\widehat{y}^{ \pm 1}$-arrows as we encounter occurrences of $y^{ \pm 1}$ (except when a $\widehat{y}^{ \pm 1}$-arrow is already defined there, which can happen below $\beta_{1}$ ). We arrange that the head of a new $y^{ \pm 1}$-arrow not coincide with its tail or with either end of any previous $y^{ \pm 1}$-arrow. We are done unless we are so unlucky that $\delta \hat{w}_{k}=\delta$. But there must be at least one $\widehat{y}^{ \pm 1}$-arrow, so if $\delta \widehat{w}_{k}=\delta$, we change the head of the last $\widehat{y}^{ \pm 1}$-arrow (without changing its tail or either end of any previous $y$-arrow, which is possible because of how we arranged the heads of the new $\widetilde{y}^{ \pm 1}$-arrows and because our choices of $\beta$ and $\delta$ guarantee that the $\widetilde{y}^{ \pm 1}$-arrow being changed is new).

\section{Free products Represented on $Q$}

The next theorem deals with countable groups which can be embedded in $A(Q)$. Equivalently ([11, Theorem 7.1.2], and [4, Theorem 4] or the present Lemma 3), these are the countable groups which can be right ordered (equipped with a total order preserved by multiplication on the right). Here we reprove a special case of $[4$, Theorem 4] so as to sharpen the conclusion a bit.

Lemma 3. Let $G$ be a countable right orderable group. Then $G$ can be embedded as a subgroup $\hat{G}$ of $A(\mathbf{Q})$ in such a way that no $e \neq \hat{g} \in \hat{G}$ fixes any point in $Q$.

Proof: Let $(G, \leqslant)$ be a right ordering of $G$, and form the right regular representation of $G$. If $(G, \leqslant)$ is dense in itself, we are done. If instead it is discrete, replace each point in $(G, \leqslant)$ by a copy of $Q$, and let $G$ act on the resulting chain in the obvious way. 
We pause to point out a few things about right orderable groups which we won't actually use here: They are torsion free and are unique product groups. Several characterisations of them are given in [11, Section 7.1]. They form a quasi-variety of groups (closed under subgroup, direct product, and ultraproduct) and thus can be characterised in first order group theoretic language.

The following proof is modelled after the proof in [3] of the corresponding theorem for lattice-ordered groups. A word of caution: Within the lattice-ordered group free product of lattice-ordered groups $G$ and $H$ which are not totally ordered, the subgroup generated by $G$ and $H$ is not their free product as groups [5, Theorem 3].

Proof of Theorem C: The proof splits into the same three phases as the proof of Theorem A.

FAITHFUlNESS AND LINKAGE. Since the free product of right orderable groups is right orderable [11, Theorem 7.3.2], we can embed $G * H$ in $A(Q)$ so that no nonidentity element fixes any point of $Q$ (Lemma 3). We so represent $G * H$ on each of the rational intervals $(2 n, 2 n+1), n \geqslant 0$, denoting the embedding by $\phi_{n}$. Later we shall modify the restriction $\phi_{n} \mid H$, but not $\phi_{n} \mid G$.

We enumerate the nonidentity elements of $G * H$ as $w_{0}, w_{1}, \ldots$ Now $w_{0} \phi_{0}$ moves some point $\alpha_{0} \in(0,1)$, and thus gives rise to a diagram with base point $\alpha_{0}$ witnessing within $(0,1)$ the fact that $w_{0} \neq e$. Let $\lambda_{0}$ denote the leftmost point of this diagram, and $\rho_{0}$ the rightmost (see Figure 5). Since no nonidentity element of $(G * H) \phi_{0}$ fixes any point in $(0,1)$, we can pick $h_{\lambda_{0}} \in H$ such that $0<\lambda_{0} h_{\lambda_{0}}<\lambda_{0}$. Similarly, we can pick $h_{\rho_{0}} \in H$ such that $\rho_{0}<\rho_{0} h_{\rho_{0}}<1$. Next we pick $f_{0} \in A((-1 / 2,3 / 2))$ such that $\left(\lambda_{0} h_{\lambda_{0}}\right) f_{0}=-1 / 4,\left(\rho_{0} h_{\rho_{0}}\right) f_{0}=5 / 4$ and $\operatorname{supp}\left(f_{0}\right) \subseteq\left(-1 / 2, \lambda_{0}\right) \cup\left(\rho_{0}, 3 / 2\right)$.

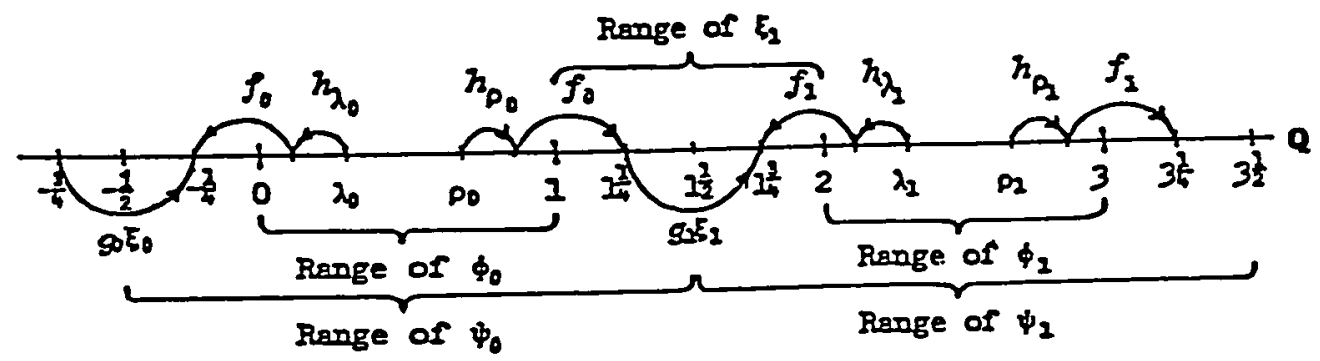

Figure 5

For $w \in H$, let $w \psi_{0}^{H}=f_{0}^{-1}\left(w \phi_{0}\right) f_{0}$. Thus $\psi_{0}^{H}$ is a representation of $H$ on $(-1 / 2,3 / 2)$. For $w \in G$, let $w \psi_{0}^{G}=w \phi_{0}$ on $(0,1)$ and let $\psi_{0}^{G}$ be the identity action on $(-1 / 2,0) \cup(1,3 / 2)$. (This identity action will be changed later.) Let $\psi_{0}$ be the 
unique extension of $\psi_{0}^{G}$ and $\psi_{0}^{H}$ to an order-preserving representation of $G * B$ on $(-1 / 2,3 / 2)$. Now $\psi_{0}$ agrees with $\phi_{0}$ at all diagram points, so that the diagram with base point $\alpha_{0}$ arising from $w_{0} \psi_{0}$ coincides with that arising from $w_{0} \phi_{0}$. Also, the orbit $\alpha_{0}\left((G * H) \psi_{0}\right)$ spills outside $(0,1)$ to include both $-1 / 4$ and $5 / 4$.

We repeat this for $n=1,2, \ldots$, obtaining representations $\psi_{n}$ of $G * H$ on $(-1 / 2+2 n, 2 n+3 / 2)$.

Now for each $n \geqslant 0$, we pick an order-preserving action $\xi_{n}$ of $G$ on $(2 n-1,2 n)$ for which $(2 n-3 / 4)\left(g_{n} \xi_{n}\right)=2 n-1 / 4$ for some $g_{n} \in G$. We splice together these various actions by defining $\psi$ to be the unique (faithful) order-preserving representation of $G * H$ on $(-1, \infty)$ such that

(1) for $w \in G, w \psi= \begin{cases}w \psi_{n} \text { on }(2 n, 2 n+1), & n \geqslant 0, \text { and } \\ w \xi_{n} \text { on }(2 n+1,2 n+2), & n \geqslant-1 ;\end{cases}$
(2) for $w \in H, w \psi=\left\{\begin{array}{l}w \psi_{n} \text { on }(2 n-1 / 2,2 n+3 / 2), \quad n \geqslant 0, \text { and } \\ e \text { on }(-1,-1 / 2) .\end{array}\right.$

Note that for each $n \geqslant 0$, the diagram with base point $\alpha_{n}$ arising from $w_{n} \psi$ coincides with that arising from the original $w_{n} \phi_{n}$, making $\psi$ faithful.

Moreover, all the points from the various diagrams (and also the points $-3 / 4$ and $-1 / 4)$ lie in the same orbit of $(G * H) \psi$. At present $H \psi$ is the identity action on $(-1,-1 / 2)$, but we change this (and define $H \psi$ also on $(-\infty,-1 / 2)$ ) by choosing $v$ in accordance with

CLAIM 1. There is a representation $v$ of $H$ on $(-\infty,-1 / 2)$ such that for each integer $m>0$ there exists $h_{m} \in H$ for which $(-m)\left(h_{m} v\right)=-m-1$ and such that $-3 / 4$ lies in the same orbit of $H v$ as do the nonnegative integers.

Proof: First pick a representation $\theta$ of $H$ on $Q$ such that $0\left(h_{1} \theta\right)<0$ for some $h_{1} \in H$. Discarding the rationals below inf $(0(H \theta))$, we may assume that inf $(0(H \theta))=$ $-\infty$, and similarly that $\sup (0(H \theta))=+\infty$. Since $\inf (0(H \theta))=-\infty$, we can pick $h_{2}, h_{3}, \cdots \in H$ such that

$$
\left(0\left(h_{1} \theta\right) \ldots\left(h_{i} \theta\right)\right)\left(h_{i+1} \theta\right)<0\left(h_{1} \theta\right) \ldots\left(h_{i} \theta\right), \quad i=1,2, \ldots,
$$

and such that this decreasing sequence of points approaches $-\infty$. Finally, transfer $\theta$ to a representation of $H$ on $(-\infty,-1 / 2)$ via a chain isomorphism $\theta_{2}$ from $Q$ onto $(-\infty,-1 / 2)$ such that

$$
\begin{cases}0 \theta_{2}=-3 / 4, & \text { and } \\ \left(0\left(h_{1} \theta\right) \ldots\left(h_{i} \theta\right)\right) \theta_{2}=-i, & i=1,2, \ldots\end{cases}
$$


Having chosen $v$ as in Claim 1 , we redefine $\psi^{H}$ on $(-\infty,-1 / 2)$ to agree with $v$, and redefine $\psi$ accordingly. (This preserves the properties of $\psi$ arranged above.) Since all diagram points lie in the same orbit of $(G * H) \psi$ as $-3 / 4$ which in turn lies in the same orbit as all negative integers, we have linkage.

HIGH ORDER-TRANSITIVITY. Let $k_{p}=\dot{h_{1}} \ldots h_{p} \in H$. We have $(-1)\left(k_{p} \psi\right)=$ $(-1)\left(k_{p} v\right)=-p-1$.

We enumerate the set of pairs of $n$-tuples $\alpha_{1}<\ldots<\alpha_{n}$ and $\beta_{1}<\ldots<\beta_{n}$ of rationals below -1 . Let $m_{0}=-1$. For the first pair $\left(\alpha_{1}, \ldots, \alpha_{n_{1}}\right),\left(\beta_{1}, \ldots, \beta_{n_{1}}\right)$, we pick $p_{1}$ large enough that $\beta_{n_{1}} k_{p_{1}}<\alpha_{1}$, and we pick an integer $m_{1}<\beta_{1}\left(k_{p_{1}} \psi\right)$. Then we use Claim 2 to pick a representation $\zeta_{1}$ of $G$ on $\left(m_{1}, m_{0}\right)$ such that $\alpha_{i}\left(g_{1} \zeta_{1}\right)=$ $\beta_{i}\left(k_{p_{1}} \psi\right), i=1, \ldots, n_{1}$, for some $g_{1} \in G$. We have $\alpha_{i}\left(g_{1} \zeta_{1}\right)\left(k_{p_{1}}^{-1} \psi\right)=\beta_{i}, i=$ $1, \ldots, n_{1}$.

CLAIM 2. Let $\sigma_{1}<\ldots<\sigma_{n}<\tau_{1}<\ldots<\tau_{n} \in Q$. Then there is a representation $\zeta$ of $G$ on $Q$ such that $\tau_{i}(g \zeta)=\sigma_{i}, i=1, \ldots, n$, for some $g \in G$.

PROOF OF CLAIM: First, pick a representation $\theta$ of $G$ on $Q$ such that $0(g \theta)<0$ for some $g \in G$. Then pick $\tau_{1}^{\prime}<\ldots<\tau_{n}^{\prime}$ strictly between $0(g \theta)$ and 0 , and let $\sigma_{i}^{\prime}=\tau_{i}^{\prime}(g \theta), i=1, \ldots, n$. Then $\sigma_{n}^{\prime}<\tau_{1}^{\prime}$. Transfer $\theta$ to a map $\zeta$ of $G$ on $Q$ via a chain automorphism of $Q$ that maps $\sigma_{i}^{\prime}$ to $\sigma_{i}$ and $\tau_{i}^{\prime}$ to $\tau_{i}, i=1, \ldots, n$.

For the next pair $\left(\mu_{1}, \ldots, \mu_{n_{2}}\right),\left(\nu_{1}, \ldots, \nu_{n_{2}}\right)$, we pick $q_{2}$ large enough that $\mu_{n_{2}}\left(k_{q_{2}} \psi\right)<m_{1}$ and then $p_{2}$ large enough that $\nu_{n_{2}}\left(k_{p_{2}} \psi\right)<\mu_{1}\left(k_{q_{2}} \psi\right)$, and we pick an integer $m_{2}<\nu_{1}\left(k_{p_{2}} \psi\right)$. We now use Claim 2 to pick a representation $\zeta_{2}$ of $G$ on $\left(m_{2}, m_{1}\right)$ such that $\mu_{i}\left(k_{q_{2}} \psi\right)\left(g_{2} \zeta_{2}\right)=\nu_{i}\left(k_{p_{2}} \psi\right), i=1, \ldots, n_{2}$, for some $g_{2} \in G$. We have

$$
\mu_{i}\left(k_{q_{2}} \psi\right)\left(g_{2} \zeta_{2}\right)\left(k_{p_{2}} \psi\right)^{-1}=\nu_{i}, \quad i=1, \ldots, n_{2} .
$$

We continue in this fashion. Then we define $\psi^{G}$ on $(-\infty,-1)$ to agree with $\zeta_{n}$ on $\left(m_{n+1}, m_{n}\right), n=1,2, \ldots$, and to preserve order.

SYNTHESIS. Finally, we define $\psi$ to be the unique (faithful, order-preserving) extension of $\psi^{G}$ and $\psi^{H}$ to a representation of $G * H$ on $Q$.

\section{FREE PRODUCTS ON $\mathrm{N}$}

Proof of Theorem D: Pick $\bar{h} \in \boldsymbol{H}$ having infinite order.

FAITHFULNESS AND LINKAGE. Let $\Delta$ be any $\langle\bar{h}\rangle$-orbit in the right regular representation of $H$. The action of $\langle\bar{h}\rangle$ on $\Delta$ is the regular representation $(Z, Z)$ of the integers. Pick $\delta \in \Delta$, and order $\Delta$ by setting $\delta \bar{h}^{m} \leqslant \delta \bar{h}^{n}$ if and only if $m \geqslant n$ (so that $\bar{h}$ moves these points downwards). Let $\mathcal{O}$ be the set of such orbits $\Delta$, including if necessary multiple pairwise disjoint copies of them in order to make $\operatorname{card}(\mathcal{O})=\aleph_{0}$. Choose any order 
of the union of the orbits (including multiple copies) in $\mathcal{O}$ which extends the orders on the individual $\Delta$ 's and produces a copy $Q$ of the rationals in which each $\Delta$ is a coset of $Z$. This gives an action $\phi^{H}$ of $H$ on $Q$ for which $\alpha\left(h \phi^{H}\right)=\alpha-1$ for all $\alpha \in Q$. This action (and the other actions of $H$ yet to be specified) will remain unchanged throughout the proof, whereas actions of $G$ will often be changed. Temporarily, let $\phi^{G}$ be the identity action of $G$ on $Q$.

Index $\mathcal{O}$ by $N \times N$ and from each $\Delta \in \mathcal{O}$ pick one special point $\delta_{\Delta} \in \Delta \cap Q^{+}$. Let $\Sigma_{i}$ consist of those special points indexed by pairs $(i, n), n \in N$.

Pick $e \neq \bar{g} \in G$.

Enumerate the nonidentity elements of $G * H$ as $w_{0}, w_{1}, \ldots$ (written in reduced form). For each $i \geqslant 0$, let $\Gamma_{i}$ be a copy of the set $G * H$ (with the $\Gamma_{i}$ 's pairwise disjoint and disjoint from $Q$ ), and let $\theta_{i}$ be the right regular representation of $G * H$ on $\Gamma_{i}$. Pick $n_{i} \geqslant 1$ sufficiently large that $(\bar{h} \bar{g})^{n_{i}}$ is not an initial subword of $w_{i}$, and let $\Lambda_{i}$ consist of those points of $\Gamma_{i}$ which end in a power $(\bar{h} \bar{g})^{n}$ of $\bar{h} \bar{g}$ with $n \geqslant n_{i}$ (the special points of $\left.\Gamma_{i}\right) \cdot \operatorname{Card}\left(\Lambda_{i}\right)=\aleph_{0}$, and we put $\Lambda_{i}$ into one-to-one correspondence with $\Sigma_{i}$.

In order to make $\Gamma_{i}$ interact with $Q$, we change the restriction $\theta_{i}^{G}=\theta_{i} \mid G$ of the action of $G * H$ on $\Gamma_{i}$ and also the identity action $\phi_{i}^{G}=\phi^{G} \mid \Sigma_{i}$ of $G$ on $\Sigma_{i}$. We do this by interchanging each special point in $\Lambda_{i} \subseteq \Gamma_{i}$ with the corresponding special point in $\Sigma_{i} \subseteq Q^{+}$. (For any one pair of corresponding special points, this interchange amounts to conjugation of $\theta_{i}^{G} \cup \phi_{i}^{G}$ by the transposition that interchanges those special points.) This new action $\psi_{i}^{G}$ of $G$ on $\Gamma_{i} \cup \Sigma_{i}$ fixes all points in $\Lambda_{i} \subseteq \Gamma_{i}$ but moves the points in $\boldsymbol{\Sigma}_{\boldsymbol{i}} \subseteq \mathrm{Q}^{+}$.

Let $\psi^{G}$ denote the union of the actions $\psi_{i}^{G}$, with $\psi^{G}$ the identity action on the nonspecial points of $Q^{+}$and (temporarily) on $Q^{-} \cup\{0\}$. This action of $G$ on $\Omega^{+}=$ $\mathrm{Q}^{+} \cup \bigcup_{i} \Gamma_{i}$ will not be further changed. Let $\psi^{H}=\phi^{H} \cup \bigcup_{i}\left(\theta_{i} \mid H\right)$. Let $\psi$ be the unique extension of $\psi^{G}$ and $\psi^{H}$ to a representation of $G * H$ on $\Omega=Q^{-} \cup\{0\} \cup \Omega^{+}=Q \cup \bigcup_{i} \Gamma_{i}$.

Write $w_{i}$ (in reduced form) as $w_{i}=w_{i 1} \ldots w_{i n}\left(w_{i j} \in G \cup H\right)$. Pick any $w_{i j}$, and let $w_{i}^{*}=w_{i j}^{*}$ be the initial subowrd of $w_{i}$ preceding $w_{i j}$. Let $e_{i}$ denote the identity element in $\Gamma_{i}$. We have $e_{i}\left(w_{i}^{*} \theta_{i}\right)=w_{i}^{*}$ because $\theta_{i}$ is just the right regular representation of $G * H$. Then

$$
e_{i}\left(w_{i}^{*} \theta_{i}\right)\left(w_{i j} \psi\right)=e_{i}\left(w_{i}^{*} \theta_{i}\right)\left(w_{i j} \theta_{i}\right)=w_{i}^{*} w_{i j}
$$

because $e_{i}\left(w_{i}^{*} \theta_{i}\right)$ and $e_{i}\left(w_{i}^{*} \theta_{i}\right)\left(w_{i j} \theta_{i}\right)$ are initial words of $w_{i}$ and so are not special elements in $\Lambda_{i} \subseteq \Gamma_{i}$. Hence

$$
e_{i}\left(w_{i} \psi\right)=e_{i}\left(w_{i} \theta_{i}\right) \neq e_{i} \text { for every } i
$$

Therefore the representation $\psi$ is faithful. 
For the linkage, we want to know that given any $\alpha_{1}, \ldots, \alpha_{n} \in \Omega^{+}$, there exists $k \in G * H$ such that all points $\alpha_{i}(k \psi)$ belong to $\mathrm{Q}^{+}$(or maybe $\mathrm{Q}^{-}$). Then since $\alpha(h \psi)=\alpha-1$ for $\alpha \in Q$, following $k \psi$ by a suitable power of $\bar{h} \psi$ will move all $\alpha_{i}$ 's into $Q^{-}$. For this, it suffices to show

CLAIM 1. Given any $\beta \in \Omega^{+}$, there exists $m_{\beta}$ such that $\beta((\bar{h} \psi)(\bar{g} \psi))^{m} \in \mathbf{Q}$ for all $m \geqslant m_{\beta}$.

Proof of Claim: First, suppose $\beta \in \Gamma_{i} \backslash \Lambda_{i}$. As we multiply $\beta \in G * H$ on the right alternately by $\bar{h}$ (first), then $\bar{g}$, then $\bar{h}$, then $\bar{g}, \ldots$, there must be a first time that we obtain an element of $\Lambda_{i}$.

If this first occurs upon multiplication by $\bar{g}$, we have $\tau \bar{h} \in \Gamma_{i} \backslash \Lambda_{i}$ but $\tau \bar{h} \bar{g} \in \Lambda_{i}$ for some $\tau=\beta(\bar{h} \bar{g})^{r}(r \geqslant 0)$, with $\beta u \in \Gamma_{i} \backslash \Lambda_{i}$ for all initial words $u$ of $(\bar{h} \bar{g})^{r}$. Then

and

$$
\beta((\bar{h} \psi)(\bar{g} \psi))^{r}=\beta\left(\left(\bar{h} \theta_{i}\right)\left(\bar{g} \theta_{i}\right)\right)^{r}=\beta(\bar{h} \bar{g})^{r}=\tau
$$

also

$$
\tau(\bar{h} \psi)=\tau\left(\bar{h} \theta_{i}\right)=\tau \bar{h}
$$

but

$$
\begin{gathered}
(\tau \bar{h})\left(\bar{g} \theta_{i}\right)=\tau \bar{h} \bar{g}, \\
(\tau \bar{h})(\bar{g} \psi)=\sigma,
\end{gathered}
$$

where $\sigma$ is the special point in $\Sigma_{i} \subseteq \mathrm{Q}^{+}$corresponding to $\tau \bar{h} \bar{g} \in \Lambda_{i}$. Further alternate applications of $\bar{h} \psi$ and $\bar{g} \psi$ keep $\sigma$ in $\mathbf{Q}$. This is because each orbit of $\langle\bar{h} \psi\rangle$ in $\mathbf{Q}$ contains just one special point, and because $\bar{g} \psi$ fixes all nonspecial points of $Q$. This establishes the claim for this case.

If the first time we obtain an element of $\Lambda_{i}$ occurs upon multiplication by $\bar{h}$ (possible because of cancellation involving $w$ ), then for some $\tau=\beta(\bar{h} \bar{g})^{r}$ we have $\tau \in \Gamma_{i} \backslash \Lambda_{i}$ but $\tau \bar{h} \in \Lambda_{i}$. We have

$$
\beta((\bar{h} \psi)(\bar{g} \psi))^{r}=\beta\left(\left(\bar{h} \theta_{i}\right)\left(\bar{g} \theta_{i}\right)\right)^{r}=\beta(\bar{h} \bar{g})^{r}=\tau .
$$

Since we did not change $\theta_{i} \mid H$,

$$
\tau(\bar{h} \psi)=\tau\left(\bar{h} \theta_{i}\right)=\tau \bar{h}
$$

Since in the original representation, $\bar{g} \psi^{G}$ fixes the special point in $\Sigma_{i}$ corresponding to $\tau \bar{h} \in \Lambda_{i}, \bar{g} \psi$ fixes $\tau \bar{h}$, so unfortunately $\tau \bar{h}(\bar{g} \psi) \notin Q$. However, we continue to apply to $\tau \bar{h}(\bar{g} \psi)$ alternately $\bar{h} \psi, \bar{g} \psi, \bar{h} \psi, \bar{g} \psi, \ldots$, which multiplies $\tau \bar{h}$ alternately by $\bar{h}, \bar{g}, \bar{h}, \bar{g}, \ldots$, until we again obtain an element of $\Lambda_{\boldsymbol{i}}$. If this occurs upon multiplication by $\bar{g}$, we proceed as first case; if it occurs upon multiplication by $\bar{h}$, we continue this process. Eventually no more cancellation with $w$ occurs. After that, if multiplication by $\bar{h}$ gives us an element of $\Lambda_{i}$, then multiplication by $\bar{g}$ gives us an element of $\Gamma_{i} \backslash \Lambda_{i}$ 
(by the definition of $\Lambda_{i}$ ), and finally the next multiplication giving us an element of $\Lambda_{i}$ must be by $\bar{g}$ (again by the definition of $\Lambda_{i}$ ), and we are done.

Next, if $\beta \in \Lambda_{i}$, then $\beta \bar{h} \in \Gamma_{i} \backslash \Lambda_{i}$ and $\beta \bar{h} \bar{g} \in \Lambda_{i}$, and we apply the first paragraph of the proof.

Finally, suppose that $\beta \in Q^{-}$, but that some $\beta((\bar{h} \psi)(\bar{g} \psi))^{r}$ lies in some $\Gamma_{i}$. By what has already been shown, $\beta((\bar{h} \psi)(\bar{g} \psi))^{r}((\bar{h} \psi)(\bar{g} \psi))^{\bullet} \in \mathbf{Q}$ for sufficiently large s.

HIGH ORDER-TRANSITIVITY. We need to specify $\psi^{G}$ on $Q^{-}$so that for any two $n$-tuples $\left(\alpha_{1}, \ldots, \alpha_{n}\right)$ and $\left(\beta_{1}, \ldots, \beta_{n}\right)$ of distinct points of $Q^{-}$, there exists $f \in G * H$ such that $\alpha_{i}(f \psi)=\beta_{i}, i=1, \ldots, n$. Clearly it suffices to treat the case in which the $\beta_{i}$ 's are distinct from the $\alpha_{i}$ 's.

We enumerate the set of pairs of such $n$-tuples. Let $m_{1}=0$. For the first pair $\left(\alpha_{1}, \ldots, \alpha_{n_{1}}\right),\left(\beta_{1}, \ldots, \beta_{n_{1}}\right)$, we pick an integer $m_{1}<\min \left\{\alpha_{1}, \ldots, \alpha_{n_{1}}, \beta_{1}, \ldots, \beta_{n_{1}}\right\}$. We use the following Claim 2 to pick a representation $\zeta_{1}$ of $G$ on $\left(m_{1}, m_{0}\right)$ such that $\alpha_{i}\left(\bar{g} \zeta_{1}\right)=\beta_{i}, i=1, \ldots, n_{1}$.

CLAIM 2. There is a representation $\zeta$ of $G$ on $\left[m_{1}, m_{0}\right)$, not necessarily orderpreserving, such that $\alpha_{i}(\bar{g} \zeta)=\beta_{i}, i=1, \ldots, n_{1}$.

ProOf of ClaIM: Partition $\left[m_{1}, m_{0}\right)$ into $n_{1}$ countable subsets $\Pi_{1}, \ldots, \Pi_{n_{1}}$, with $\alpha_{i}, \beta_{i} \in \Pi_{i}$. In the right regular representation $(G, G)$ of $G, \bar{g}$ moves some $\alpha$ to some $\beta \neq \alpha$. For each $i=1, \ldots, n_{1}$, establish a correspondence between the (finite or countable) set $G$ and some subset of $\Pi_{i}$ such that $\alpha \leftrightarrow \alpha_{i}$ and $\beta \leftrightarrow \beta_{i}$. Use these correspondences to transfer $n_{1}$ copies of $(G, G)$ to obtain the desired $\zeta$ (letting the action be the identity on points of $\left[m_{1}, m_{0}\right)$ not involved in the correspondences).

For the second pair $\left(\mu_{1}, \ldots, \mu_{n_{2}}\right),\left(\nu_{1}, \ldots, \nu_{n_{2}}\right)$, we pick

$$
m_{2}<\min \left\{\mu_{1}-m_{1}, \ldots, \mu_{n_{2}}-m_{1}, \nu_{1}-m_{1}, \ldots, \nu_{n_{2}}-m_{1}\right\},
$$

and use Claim 2 to pick a representation $\zeta_{2}$ of $G$ on $\left[m_{2}, m_{1}\right)$ for which

$$
\left(\mu_{i}-m_{1}\right)\left(\bar{g} \zeta_{2}\right)=\nu_{i}-m_{1}, \quad i=1, \ldots, n_{2} \text {. }
$$

Then we have

$$
\mu_{i}(\bar{h} \psi)^{m_{1}}\left(\bar{g} \zeta_{2}\right)(\bar{h} \psi)^{-m_{1}}=\nu_{i}, \quad i=1, \ldots, n_{2} .
$$

We continue in this fashion. Then we define $\psi^{G}$ so that for all $g \in G, g \psi^{G} \equiv g \zeta_{i}$ on $\left[m_{i}, m_{i-1}\right), i=1,2,3, \ldots$, and so that $g \psi^{G}$ acts as before on $\Omega^{+}$.

SYNThEsis. Finally, we let $\psi$ be the unique (faithful, highly transitive) representation of $G * H$ on $\Omega$ which extends $\psi^{G}$ and $\psi^{H}$.

Not every free product of groups which are at most countable can be represented as in Theorem D. Specifically, $Z_{2} * Z_{2}$ cannot: 
Proposition 4. Let $G$ be a group generated by two elements of order two. Then $G$ has no doubly transitive representation on $N$, not even a nonfaithful representation.

Proof: Let $G=\langle g, h\rangle$, with $g$ and $h$ of order 2. Suppose by way of contradiction that $\widehat{G}$ is a doubly transitive representation of $G$ on $N$. The permutation $\widehat{g}$ consists of transformations $\left(1,1^{\prime}\right),\left(2,2^{\prime}\right), \ldots$, and (maybe) fixed points, as does $\widehat{h}$.

Consider first the case in which $\widehat{g}$ has no fixed points.

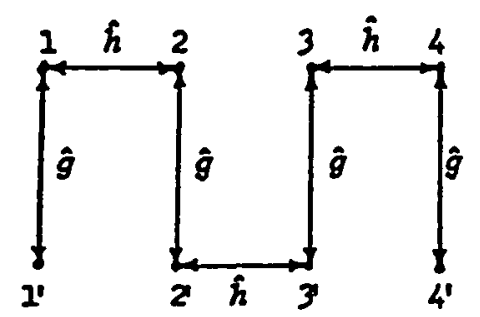

Figure 6. The cycles of $\widehat{g}$.

We trace a "path" $P$ of points beginning at 1 . Unless $1 \widehat{h}=1$, so that the path terminates with only the one point 1 , the next point is "new" (not 1 or $1^{\prime}$ ) because $\widehat{G}$ has no proper fixed blocks. With no loss of generality, $1 \widehat{h}=2$. Unless $2^{\prime} \widehat{h}=2^{\prime}$, causing termination, $2^{\prime} \widehat{h}$ must be new (not 1 or 2 because $\widehat{h}$ is one-to-one, not $1^{\prime}$ because $\left\{1,1^{\prime}, 2,2^{\prime}\right\}$ would be a fixed block of $\left.\widehat{G}\right)$. With no loss of generality, $2^{\prime} \widehat{h}=3^{\prime}$. Again unless $3 \widehat{h}=3,3 \widehat{h}$ must be new (not $1,1^{\prime}, 2,2^{\prime}, 3,3^{\prime}$ ). Continuing in this fashion, we obtain a (possibly finite) set $P$ of points.

We trace a similar path $P^{\prime}$ starting at $\mathbf{1}^{\prime} . P$ and $P^{\prime}$ have no point in common because $\widehat{h}$ is one-to-one. Let the points of $P^{\prime}$ be $1^{\prime}, n_{1}^{\prime}, n_{1}, n_{2}, n_{2}^{\prime}, \ldots$ (perhaps terminating). Then $P \cup P^{\prime}$ is a fixed block of $N$ and thus all of $N$, and the set of primed points of $\mathrm{N}$ and the set of unprimed points of $\mathrm{N}$ both form blocks of $\widehat{G}$, in violation of double transitivity.

Now suppose $\widehat{g}$ has at least one fixed point $\alpha$.

We trace a path $P$ beginning at $\alpha$. Now $\alpha \widehat{h}$ cannot be fixed by $\widehat{g}$, and with no loss of generality $\alpha \widehat{h}=1$, the next point in $P$. Also $1^{\prime} \widehat{h}$ must be new (not $\left.\alpha, 1,1^{\prime}\right)$ and not fixed by $\widehat{g}$; with no loss of generality $1^{\prime} \hat{h}=2$. This infinite path $P$, being a block of $\widehat{G}$, must be all of $N$ (so that $\alpha$ is the only fixed point of $\widehat{g}$ ). But the action of $\langle\widehat{g}, \widehat{h}\rangle$ on $P$ is not doubly transitive-since every $w \in G$ is a string of alternating $\hat{g}$ 's and $\widehat{h}$ 's, only $e$ and $\widehat{g}$ fix $\alpha$. 


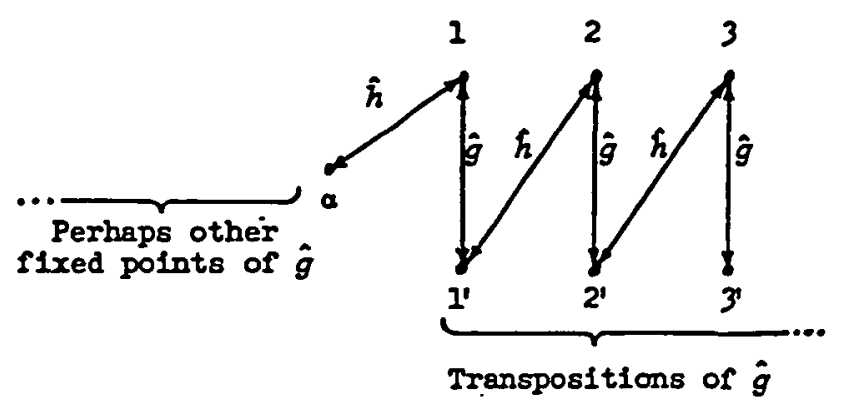

Figure 7. The cycles of $\widehat{g}$.

\section{Free LATTICE-ORDERED GROUPS}

Many of the ideas in the preceding proofs came from earlier work by the authors on free lattice-ordered groups. Here we give a brief description of the free $\ell$-group $L_{\eta}$ of rank $\eta$, followed by some new results about free $\ell$-groups which grew out of our research on free groups, completing the circle. The expository article [0] is a good source of further information, and familarity with it is assumed in the proofs.

In the free $\ell$-group $L_{\eta}$ on a set $\mathbf{x}$, the subgroup generataed by $\mathbf{x}$ is a copy of $F_{\eta}$. Each $w \in L_{\eta}$ can be expressed as $w=\bigvee_{i} \bigwedge_{j} w_{i j}$, a finite supremum of finite infima of group words (though this expression is far from unique).

For $2 \leqslant \eta \leqslant \aleph_{0}, L_{\eta}$ can be faithfully represented as a highly order-transitive l-permutation group $\widehat{L}_{\eta}$ on $\mathbf{Q}$, meaning that $\widehat{L}_{\eta}$ is an $\ell$-group of order-preserving permutations of $Q$ under the pointwise order

$$
\left\{\begin{array}{l}
\alpha(f \vee g)=\max \{\alpha f, \alpha g\}, \\
\alpha(f \wedge g)=\min \{\alpha f, \alpha g\}
\end{array}\right.
$$

The orbits $\alpha \widehat{F}_{\eta}$ and $\alpha \widehat{L}_{\eta}$ must coincide, so $\widehat{F}_{\eta}$ must be transitive on $\mathbf{Q}$. Must $\widehat{F}_{\eta}$ (can $\widehat{F}_{\eta}$ ) be highly order-transitive? The range of possible behaviours is extreme:

THEOREM 5. $L_{\eta}\left(2 \leqslant \eta \leqslant \aleph_{0}\right)$ can be faithfully represented as a highly ordertransitive $\ell$-permutation group $\widehat{L}_{\eta}$ on $Q$ in each of the following fashions:

(1) $\widehat{F}_{\eta}$ is highly transitive on $Q$ (and it can be further arranged that Theorem A4 hold for $\widehat{F}_{\eta}$ ).

(2) $\widehat{F}_{\eta}$ is uniquely transitive on $Q$.

PROOF: For the first part of (1), proceed as in the proof of Theorem A, but arrange the faithfulness of $\widehat{L}_{\eta}$ by including in the enumeration a diagram (see [9]) for each $\ell$ group word. 
To prove the rest of (1), we elaborate on the proof of Theorem A4. We arrange high order-transitivity as in that proof (but setting $\alpha \hat{t}=\alpha-1$ only for $\alpha \leqslant 0$ ), and arrange faithfulness as in the preceding paragraph. This time we include $\hat{t}$-arrows in $\mathcal{P}$. At the beginning of the induction, the only arrows having ends in $T$ are $\hat{t}$-arrows, so there are no loops involving points in $T$; we need to avoid ever creating a first such loop. This can be done as before, since even for $\hat{t}$-arrows there must exist $\lambda$ and $\rho$ as before whenever a new $\hat{t}$-arrow actually needs to be defined.

(2) follows from [8, Theorem 3], which says that $F_{\eta}$ has a right ordering $\left(F_{\eta}, \leqslant\right)$ which gives a chain isomorphic to $Q$ and for which the right regular representation of $F_{\eta}$ can be extended to a faithful highly order-transitive representation of $L_{\eta}$.

Added in Proof: Steven Gunhouse has recently proved that if $G$ and $H$ are countable or finite, then $G * H$ has a faithful highly transitive representation unless $G=H=$ $Z_{2}$. This, and most of the results of this paper, have been independently obtained by Kenneth K. Hickin (unpublished) by completely different techniques.

\section{REFERENCES}

[1] J.D. Dixon, 'Most finitely generated permutation groups are free', Bull. London Math. Soc. 22 (1990), 222-226.

[2] L. Fuchs, Partially ordered algebraic systems (Pergamon Press, New York, 1963).

[3] A.M.W. Glass, 'Free products of lattice-ordered groups', Proc. Amer. Math. Soc. 101 (1987), 11-16.

[4] W.C. Holland, 'The lattice-ordered group of automorphisms of an ordered set', Michigan Math. J. 10 (1963), 399-408.

[5] W.C. Holland, 'Group equations which hold in lattice-ordered groups', Sympos. Math. 21 (1977), 365-378.

[6] S.H. McCleary, 'O-primitive ordered permutation groups II', Pacific J. Math. 49 (1973), 431-443.

[7] S.H. McCleary, 'Free lattice-ordered groups represented as 0 -2-transitive $\ell$-permutation groups', Trans. Amer. Math. Soc. 290 (1985), 69-79.

[8] S.H. McCleary, 'An even better representation for free lattice-ordered groups', Trans. Amer. Math. Soc. 290 (1985), 81-100.

[9] S.H. McCleary, 'Free lattice-ordered groups', in Lattice-ordered groups: advances and techniques, Editors A.M.W. Glass and W.C. Holland, pp. 206-227 (Kluwer Academic Publishers, Dordrecht, 1989).

[10] T.P. McDonough, 'A permutation representation of a free group', Quart. J. Math. Oxford Ser 228 (1977), 353-356.

[11] R.B. Mura and A. Rhemtulla, Orderable groups (Marcel Dekker, New York, 1977).

[12] S. White, 'The group generated by $x \mapsto x+1$ and $x \mapsto x^{p}$ is free', J. Algebra 118 (1988), 408-422. 
Mathematics and Statistics Department

Bowling Green State University

Bowling Green, Ohio 43403

United States of America 